\title{
Ovarian cancer initially presenting with supra-clavicular lymph node metastasis: A case report
}

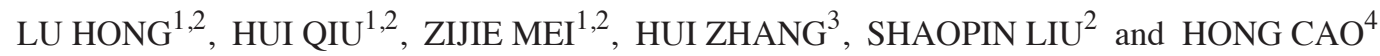 \\ ${ }^{1}$ Department of Oncology, Zhongnan Hospital of Wuhan University; ${ }^{2}$ Hubei Cancer Clinical Study Center and \\ Hubei Key Laboratory of Tumor Biological Behavior, Zhongnan Hospital of Wuhan University, Wuhan, Hubei 430071; \\ ${ }^{3}$ Department of Oncology, Ezhou Central Hospital, Ezhou, Hubei 436000; ${ }^{4}$ Department of Pathology, \\ Zhongnan Hospital of Wuhan University, Wuhan, Hubei 430071, P.R. China
}

Received November 17, 2017; Accepted April 26, 2018

DOI: $10.3892 / 01.2018 .8664$

\begin{abstract}
Supra-clavicular lymph node metastasis from ovarian cancer is rare. The present study reports the unique case of a 43-years-old patient who initially presented with left supra-clavicular lymph node metastasis, and was nulliparous. According to fine-needle aspiration cytology of the metastatic left supra-clavicular lymph nodes and $18 \mathrm{~F}$-fluorodeoxyglucose-positron emission tomography/computed tomography (PET/CT) scan, the patient was diagnosed with poorly differentiated serous carcinoma of ovarian cancer, stage IV. Following two cycles of platinum-based chemotherapy, PET/CT showed that the patient had achieved a complete response. Next, cytoreductive surgery was performed and the postoperative histological findings showed that the patient had achieved a complete pathological response. According to NCCN Clinical Practice Guidelines in Oncology, the patient was stage IV and four cycles of chemotherapy were provided, with no recurrence observed during 3 years of follow-up. This case indicates that patients who initially present with distant metastatic lymph nodes and are nulliparous might have a better prognosis and avoid overtreatment.
\end{abstract}

\section{Introduction}

Approximately $75 \%$ of patients with ovarian cancer are diagnosed with advanced disease, namely, International Federation of Gynecology and Obstetrics (FIGO) stage III-IV disease (1). Ovarian cancer metastasizes mainly through direct extension and abdominal implantation, followed by use of lymphatic channels, particularly the intra-peritoneal route, so that most

Correspondence to: Miss Hui Qiu, Department of Oncology, Zhongnan Hospital of Wuhan University, 169 Donghu Road, Wuhan, Hubei 430071, P.R. China

E-mail: qiuhui9255@163.com

Key words: supra-clavicular metastasis, ovarian cancer, complete response advanced ovarian cancer is confined in the abdominal and pelvic cavity $(2,3)$. Distant metastasis occurs in patients with stage IV disease, mainly through the lymphatic channels and via hematogenous dissemination, and these metastatic sites can be the lungs, liver, brain and distant lymph node. Distant metastatic lymph nodes are rare in the primary presence of ovarian cancer (4). Only a few cases of supra-clavicular, axillary, mediastinal and inguinal lymph node metastasis have been reported (5-8).

The standard treatment for ovarian cancer is surgical staging and maximal cytoreduction, with adjuvant platinum and taxane combination chemotherapy. For patients with stage III to IV disease whose tumor is too large to be treated surgically, neoadjuvant chemotherapy (NACT) may be considered as the primary treatment. No more than four cycles of NACT and interval debulking surgery can be a good option for these patients. A new radiological study should be performed following every two cycles of NACT. A complete pathological response ( $\mathrm{pCR}$ ) is uncommon in patients who have received chemotherapy, so is uncommon in ovarian cancer. Only a small percentage of patients $(6.5 \%)$ achieve a pathologic complete response (pCR) following NACT $(9,10)$. Furthermore, $\mathrm{pCR}$ in patients with advanced ovarian cancer receiving NACT is associated with longer progression-free survival and overall survival times compared with those in women with no pCR (10).

The present study reports the case of a patient who initially presented with metastatic left supra-clavicular lymph nodes from ovarian cancer and achieved a pCR following two cycles of NACT.

\section{Case report}

A 43-year-old woman was admitted to the Department of Radiotherapy and Chemotherapy (Gynecological Oncology) in Zhongnan Hospital of Wuhan University (Wuhan, China) in January 2014, with complaints of several enlarged left supra-clavicular lymph nodes that had been apparent for 1 week. A number of the enlarged lymph nodes were tender. Chest computed tomography (CT) showed no abnormality in the bilateral lungs, but a magnetic resonance imaging scan of the pelvis showed gross masses. Fine-needle aspiration cytology 
of the lymphadenopathy was obtained, which showed poorly differentiated carcinoma, and the metastatic supra-clavicular lymph nodes were revealed to be ovarian cancer according to the following immunohistochemical staining results: Activin receptor-like kinase 1-negative, carbonic anhydrase 9-negative, cluster of differentiation 30-negative, homeobox protein CDX-2-negative, chromogranin A-negative, cytokeratin (CK)20-negative, CK7-postive, Epstein-Barr encoding region (in situ hybridization)-negative, Ki-67-positive (90\%), paired box protein Pax-2 (PAX2)-positive, PAX8-positive, synaptophysin-negative and thyroid transcription factor 1-negative. For Immunohistochemical staining, the fixative was $4 \%$ paraformaldehyde, at room temperature, for $24 \mathrm{~h}$. The resin was paraffin and the thickness of sections was $4 \mu \mathrm{m}$. The blocking reagent was $10 \%$ goat serum (OriGene Technologies, Inc., Beijing China), for $30 \mathrm{~min}$ at room temperature. The details of primary and secondary antibody are presented in Table I. All the sections were analyzed under a phase-contrast positive microscope (Eclipse 80i; Nikon Corporation, Tokyo, Japan). Further immunostaining for Wilms tumor 1, tumor protein $\mathrm{p} 53$, p16, cancer antigen 125 (CA125), creatine kinase, estrogen receptor, postmeiotic segregation increased 2 protein, mutS homolog 2 protein, mutL homolog 1 protein and mutS homolog 6 protein was positive, while immunostaining for aspartic proteinases $\mathrm{A}$ and progestogen receptor was negative. These results showed that the metastatic supra-clavicular lymph nodes were poorly differentiated serous carcinoma (Fig. 1). At the same time, 18F-fluorodeoxyglucose-positron emission tomography/CT (FDG-PET/CT) scan revealed increased FDG uptake in the bilateral adnexal areas and in multiple lymph nodes (left supra-clavicular, mediastinal, retroperitoneal and pelvic) (Figs. 2 and 3). The CA125 level was $290.4 \mathrm{U} / \mathrm{ml}$ (normal range, 0-35 U/ml) prior to treatment. In addition, although the patient had undergone in-vitro fertilization twice, no success had been obtained, and the patient's menstrual cycle was $28 / 3$ days, which means the first day of the period follows the first day of the preceding period by 28 days, and the duration of flow is 3 days.

The patient was stage IV by FIGO staging system (1) and a complete resection was difficult, so NACT was planned as the primary treatment followed by interval debulking surgery. The NACT regimen was a platinum and taxane combination (260 mg paclitaxel and $850 \mathrm{mg}$ carboplatin, every 3 weeks). The CA125 level following the first cycle was 41.0 and the second cycle was $14.6 \mathrm{U} / \mathrm{ml}$. The CA125 level decreased to normal following the second cycle of NACT. PET/CT was used for follow-up imaging subsequent to two cycles of NACT, and it showed no markedly increased FDG uptake in the areas that were abnormal on the first PET/CT scan (Figs. 4 and 5). The patient achieved a complete response (CR) following two cycles of NACT. Next, the patient was managed with surgery plus hyperthermic intraoperative interperitoneal chemotherapy (HIPEC). The surgical therapy included a total laparoscopic hysterectomy, a bilateral salpingo-oophorectomy, an abdominal wall lesionectomy, an omentectomy, and a pelvic and para-aortic lymphadenectomy. Intraoperative views concluded that the uterus and bilateral adnexa were normal, that a small amount of ascites was present in the pelvic cavity, that there was no evident abnormality in the bowel, the large omentum, on the surface of the liver and spleen, and that only four small lesions
Table I. Details of primary and secondary antibody.

\begin{tabular}{llc}
\hline The primary antibody & \multicolumn{1}{c}{$\begin{array}{c}\text { Catalog } \\
\text { no. }\end{array}$} & Dilution \\
\hline Activin receptor-like kinase 1 & ZM-0248 & $1: 100$ \\
Carbonic anhydrase 9 & TA336805 & $1: 200$ \\
Cluster of differentiation 30 & ZA-0591 & $1: 100$ \\
Homeobox protein CDX-2 & ZA-0520 & $1: 100$ \\
Chromogranin A & ZM-0076 & $1: 100$ \\
CK20 & ZA-0574 & $1: 100$ \\
CK7 & ZM-0071 & $1: 100$ \\
Epstein-Barr encoding region & ZM-0105 & $1: 100$ \\
(in situ hybridization) & & \\
Ki-67 & ZM-0167 & $1: 25$ \\
PAX2 & ZA-0467 & $1: 20$ \\
PAX8 & ZM-0468 & $1: 50$ \\
Synaptophysin & ZM-0246 & $1: 100$ \\
Thyroid transcription factor 1 & ZM-0270 & $1: 100$ \\
Wilms tumor 1 & ZM-0269 & $1: 50$ \\
Tumor protein p53 & ZA-0501 & $1: 100$ \\
Tumor protein p16 & ZM-0205 & $1: 100$ \\
CA125 & ZM-0019 & $1: 50$ \\
Creatine kinase & ZM-0069 & $1: 100$ \\
Estrogen receptor & ZA-0102 & $1: 50$ \\
Postmeiotic segregation increased & ZA-0542 & $1: 20$ \\
2 protein & & \\
MutS homolog 2 protein & ZA-0622 & $1: 100$ \\
MutL homolog 1 protein & ZM-0154 & $1: 10$ \\
MutS homolog 6 protein & ZA-0541 & $1: 100$ \\
Aspartic proteinases A & ZM-0473 & $1: 100$ \\
Progestogen receptor & ZA-0255 & $1: 100$ \\
\hline & & \\
\hline & &
\end{tabular}

All the primary antibodies were provided by OriGene Technologies, Inc. (Beijing China), and incubated for $20 \mathrm{~min}$ at $3-8^{\circ} \mathrm{C}$. The secondary antibody was a Bond Polymer Refine Detection goat anti-rabbit poly-horseradish peroxidase-conjugated antibody (catalog number: DS9800; Leica Microsystems, Inc., Buffalo Gove, IL, USA), and was incubated for $40 \mathrm{~min}$ at room temperature at a ready-to-use dilution.

were in the right lower abdominal wall. All specimens were sent for histopathological examination. Intraoperative rapid frozen section (frozen at $-20^{\circ} \mathrm{C}$, thickness was $5 \mu \mathrm{m}$; observed under a phase-contrast positive microscope at room temperature) indicated a lack of malignant cells. Next, lobaplatin $(50 \mathrm{mg})$ was administered, at a temperature ranging from $42-43^{\circ} \mathrm{C}$, for $60 \mathrm{~min}$. The second HIPEC with same dose and temperature as the first HIPEC was completed 3 days later. Final histological findings detected no malignant cells in the bilateral adnexa or the omentum, and the pelvic and para-aortic lymph nodes were confirmed to exhibit only chronic inflammation (Fig. 6). pCR was confirmed according to the postoperative histological findings. The patient was administered another four cycles of chemotherapy ( $270 \mathrm{mg}$ paclitaxel and $120 \mathrm{mg}$ nedaplatin, every 3 weeks). No recurrence was determined during 3 years of follow-up. 


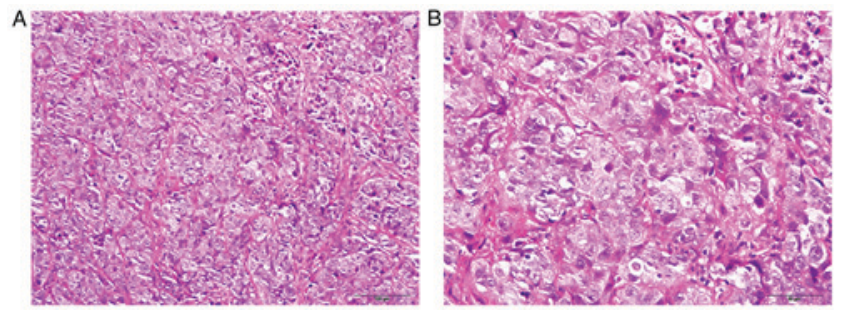

Figure 1. Fine-needle aspiration cytology of the left supra-clavicular lymph nodes showing poorly differentiated serous carcinoma from ovarian cancer. (A) HE stain; magnification, x200. (B) HE stain; magnification, x400. HE, hematoxylin and eosin
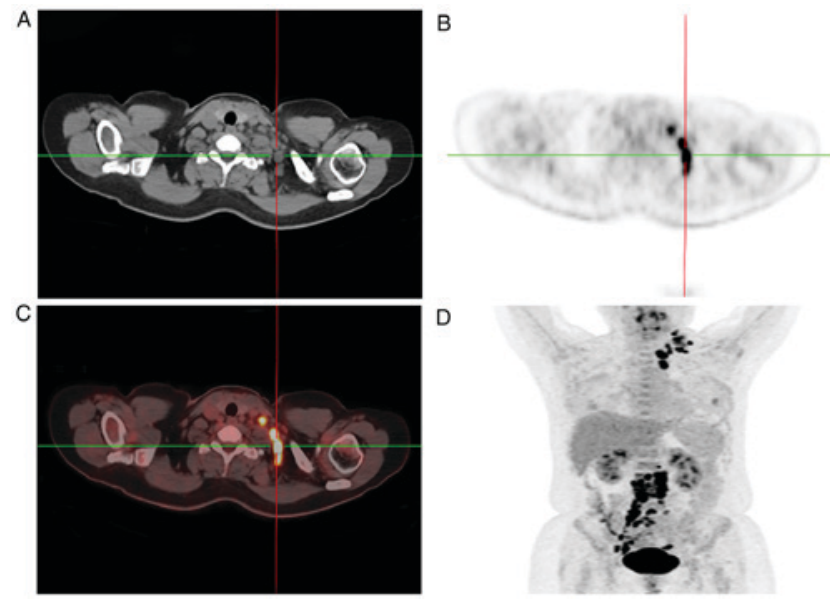

Figure 2. PET/CT scan of the left supra-clavicular lymph nodes prior to chemotherapy. (A) CT image; (B) PET image; (C) PET/CT fusion image; and (D) coronal image. PET/CT, positron emission tomography/computed tomography.
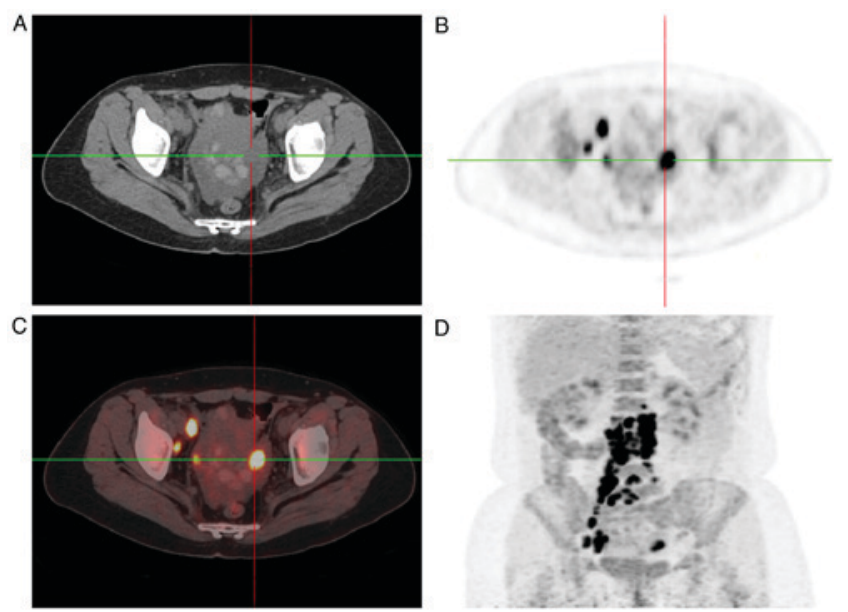

Figure 3. PET/CT scan of masses in the pelvis prior to chemotherapy. (A) CT image; (B) PET image; (C) PET/CT fusion image; and (D) coronal image. $\mathrm{PET} / \mathrm{CT}$, positron emission tomography/computed tomography.

\section{Discussion}

Ovarian cancer is caused by a variety of factors, including genetic, environment and reproductive factors. One of the known risks of ovarian cancer is being childless; women who give birth multiple times have an $8 \%$ reduction in risk for each

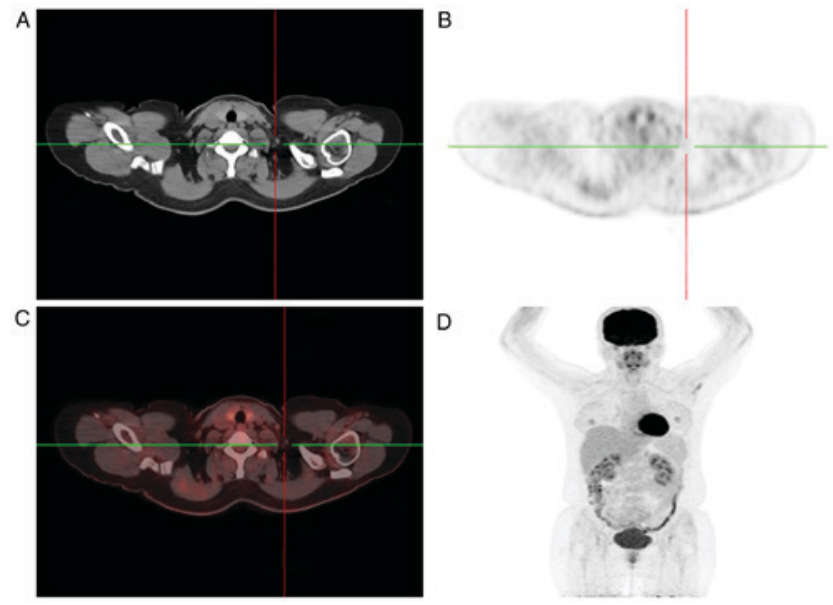

Figure 4. PET/CT scan of a lack of evident increased 18F-fluorodeoxyglucose uptake in the left supra-clavicular lymph nodes following chemotherapy. (A) CT image; (B) PET image; (C) PET/CT fusion image; and (D) coronal image. $\mathrm{PET} / \mathrm{CT}$, positron emission tomography/computed tomography.
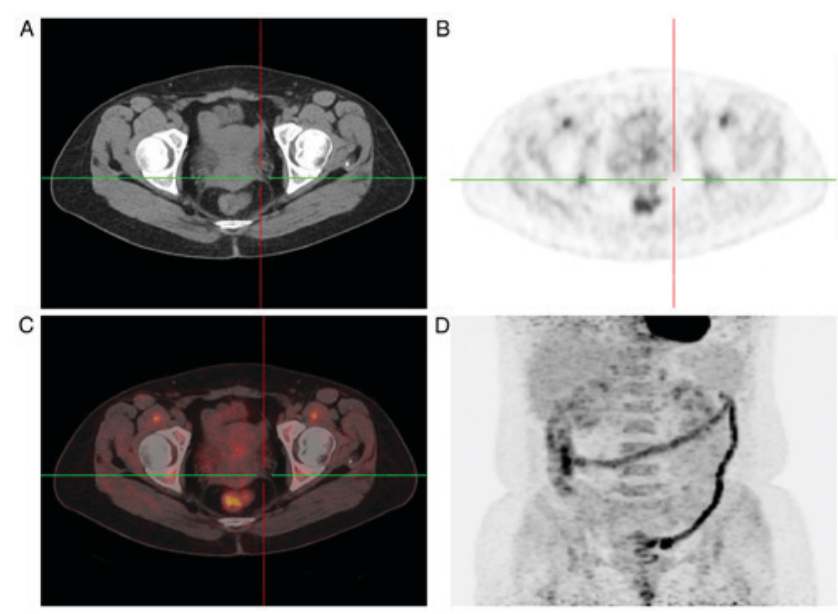

Figure 5.PET/CT scan of a lack of evidently increased 18F-fluorodeoxyglucose uptake in the pelvis following chemotherapy. (A) CT image; (B) PET image; (C) PET/CT fusion image; and (D) coronal image. PET/CT, positron emission tomography/computed tomography.
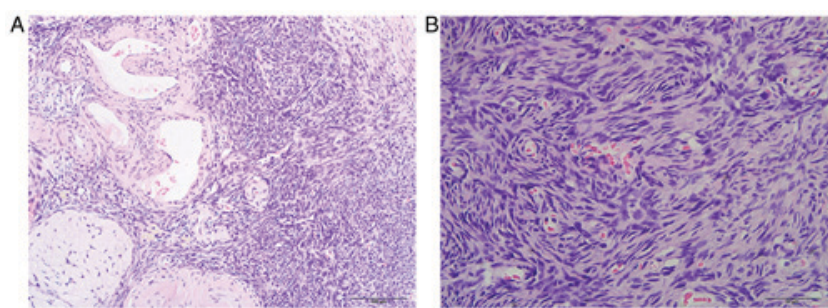

Figure 6. Specimens from ovarian surgery following chemotherapy showing (A) the corpus albicans of the ovary (HE stain; magnification, x200) and (B) normal ovarian tissues (HE stain; magnification, $x 400$ ). HE, hematoxylin and eosin.

additional pregnancy compared with nulliparous women (11). The patient in the present study was not able to become pregnant despite undergoing IVF twice.

Ovarian cancer is confined to the intraperitoneal route of dissemination by the direct exfoliation of malignant cells. The 
Table II. Ovarian cancer with distant metastatic lymph nodes reported in the literature.

\begin{tabular}{|c|c|c|c|c|c|c|c|}
\hline \multirow{2}{*}{$\begin{array}{l}\text { First } \\
\text { author } \\
\text { (year) }\end{array}$} & \multirow{2}{*}{$\begin{array}{l}\text { Age, } \\
\text { years }\end{array}$} & \multicolumn{3}{|c|}{ Distant lymph node } & \multirow{2}{*}{$\begin{array}{l}\text { Site of } \\
\text { primary } \\
\text { lesion }\end{array}$} & \multirow[b]{2}{*}{ Pathology } & \multirow[b]{2}{*}{ (Refs.) } \\
\hline & & Site & Side & Time & & & \\
\hline Cebesoy et al (2008) & - & Supra-clavicular & Left & The time of diagnosis & - & Serous & (13) \\
\hline Rahman et al (2012) & 49 & Supra-clavicular & Left & The time of diagnosis & - & Serous & (5) \\
\hline \multirow[t]{2}{*}{ Fanti et al (2006) } & 51 & Supra-clavicular & Left & After initial surgery & Left & $\begin{array}{l}\text { Poorly } \\
\text { differentiated }\end{array}$ & (14) \\
\hline & 65 & Supra-clavicular & Left & The time of diagnosis & - & $\begin{array}{l}\text { Poorly differentiated } \\
\text { serous-papillary }\end{array}$ & \\
\hline Ceccarelli et al (2011) & 48 & Axillary & Right & The time of diagnosis & Right & $\begin{array}{l}\text { Poorly differentiated } \\
\text { serous-papillary }\end{array}$ & (6) \\
\hline Hockstein et al (1997) & 78 & Axillary & Right & The time of diagnosis & Bilateral & Adenocarcinoma & (15) \\
\hline Saxena et al (2014) & 45 & Axillary & Right & The time of diagnosis & Right & Serous & $(16)$ \\
\hline Sibio et al (2014) & 49 & Axillary & - & The time of diagnosis & - & Serous papillary & (17) \\
\hline Singer et al (2001) & $\begin{array}{l}46 \\
63 \\
68\end{array}$ & $\begin{array}{l}\text { Axillary } \\
\text { Axillary } \\
\text { Axillary }\end{array}$ & $\begin{array}{l}\text { Right } \\
\text { Left } \\
\quad-\end{array}$ & $\begin{array}{l}15 \text { years after OC } \\
\text { Several years after OC } \\
\text { Several years after OC }\end{array}$ & $\begin{array}{c}\text { Bilateral } \\
\text { Bilateral } \\
-\end{array}$ & $\begin{array}{l}\text { Serous papillary } \\
\text { Serous papillary } \\
\text { Serous papillary }\end{array}$ & $(18)$ \\
\hline Aydin et al (2009) & 47 & Axillary & - & Two years after surgery & - & $\begin{array}{l}\text { Intermediate } \\
\text { differentiated serous }\end{array}$ & (19) \\
\hline Ozmen et al (2007) & $\begin{array}{l}74 \\
38\end{array}$ & $\begin{array}{l}\text { Axillary } \\
\text { Axillary }\end{array}$ & $\begin{array}{l}\text { Right } \\
\text { Right }\end{array}$ & $\begin{array}{l}4 \text { years after } \mathrm{OC} \\
2 \text { years after } \mathrm{OC}\end{array}$ & $\begin{array}{l}- \\
-\end{array}$ & $\begin{array}{l}\text { Serous papillary } \\
\text { Papillary }\end{array}$ & (20) \\
\hline Skagias et al (2008) & 63 & Axillary & Right & Several years after OC & - & $\begin{array}{l}\text { Poorly } \\
\text { differentiated }\end{array}$ & $(21)$ \\
\hline Orris et al (1999) & 63 & Axillary & Bilateral & Several years after OC & - & Adenocarcinoma & $(22)$ \\
\hline Ang et al (2007) & 59 & Inguinal & Right & The time of diagnosis & Left & $\begin{array}{l}\text { Moderately } \\
\text { differentiated } \\
\text { papillary serous }\end{array}$ & (23) \\
\hline Yang et al (2014) & 54 & Inguinal & Right & The time of diagnosis & Right & $\begin{array}{l}\text { Low-grade } \\
\text { differentiated } \\
\text { serous papillary }\end{array}$ & (8) \\
\hline
\end{tabular}

OC, ovarian cancer.

cancer can also metastasize through the lymphatic channels and the hematogenous route to the retroperitoneal and extra-peritoneal lymph nodes and other distant sites, including the lungs and bones $(4,12)$. Distant metastatic lymph nodes can occur at the time of diagnosis of ovarian cancer or during its evolution.

Ovarian cancer with metastatic lymph nodes is stage IV disease and the prognosis of these patients is naturally poor. Data from the literature concerning distant metastases are scarce, and much less is known about the effect of treatment and the prognosis of these patients compared with stage IV patients without distant metastases. A CR has rarely been reported in cases of ovarian cancer with metastatic lymph nodes. A review of the literature on ovarian cancer with distant metastatic lymph nodes, including supra-clavicular $(5,13,14)$, axillary $(6,15-22)$, and inguinal $(8,23)$ lymph node metastasis, is shown in Table II. These reports all emphasized how rare these diseases were, and how to correctly diagnose and provide them with appropriate systemic treatment. The effects of the treatments in these reports were not distinctive from the ordinary measures. Cormio et al (12) analyzed 50 patients with distant disease from 162 patients with epithelial ovarian carcinoma. Only 13 patients presented with distant metastatic disease at the time of diagnosis and not more than 5 patients had metastatic extra-abdominal lymph nodes. The study finally concluded that the duration between the ovarian cancer diagnosis and the documentation of the distant metastasis was the most important prognostic factor associated with survival, and that the survival time was longer when this duration was longer. Cheng et al (7) reported a retrospective study of 20 cases of epithelial ovarian carcinoma with extra-abdominal metastases and 645 cases without extra-abdominal metastases. Only 2 patients presented initially with extra-abdominal metastases at the time of diagnosis, and not more than 3 patients had metastatic extra-abdominal lymph nodes. In conclusion, this study indicated that the Karnofsky performance status (KPS) score, sensitivity of primary chemotherapy, metastatic site and systemic therapy following 
the diagnosis of extra-abdominal metastases were the factors that were significantly associated with survival. Zang et al (24) reviewed 25 patients with epithelial ovarian cancer who were diagnosed with initial extra-abdominal metastases. The study demonstrated that the prognosis of patients with supraclavicular lymphadenopathy or malignant pleural effusion was improved compared with that for other stage IV patients with epithelial ovarian cancer. The present report may indicate improved treatment effects and a longer survival time compared with other patients with stage IV disease from the aforementioned study $(7,24)$, with a KPS score of 1 , left supra-clavicular metastatic lymph nodes and active chemotherapy. In fact, subsequent to two cycles of chemotherapy, the patient achieved CR. Intraoperative examination and histopathological examination were negative for disease, as was the histopathological examination following the surgery.

When a patient presents with metastatic lymph nodes, it is crucial to locate the primary tumor and begin active treatment. Due to the use of PET/CT, it is easy to locate the exact origin of metastatic diseases. CR has rarely been achieved following chemotherapy for ovarian cancer, but it is possible. In the present case, the patient presented with distant metastatic lymph nodes and was nulliparous. CR was achieved following two cycles of NACT. Thus, the authors speculate whether patients who initially present with distant metastatic lymph nodes and are nulliparous, as in the present case, experience an improved prognosis and also avoid overtreatment compared with patients presenting differently. Further studies are required to confirm this and to explain the reason why such patients have a better prognosis.

\section{Acknowledgements}

Not applicable.

\section{Funding}

No funding was received.

\section{Availability of data and materials}

All data generated or analyzed during this case are included in this published article.

\section{Authors' contributions}

HQ, ZM and HZ analyzed and interpreted the patient data. SL and $\mathrm{HC}$ performed the histological and immunohistochemical of all the operative specimens. LH was a major contributor in writing the manuscript, analyzing and interpreting the patient data, gave final approval of the version to be published, and agreed to be accountable for all aspects of the article in ensuring that questions related to the accuracy. HQ performed critical revisions of the manuscript. All authors read and approved the final manuscript.

\section{Ethics approval and consent to participate}

Ethics approval and consent to participate were authorized by the ethical committee of Zhongnan Hospital of Wuhan
University (approval no. 2017060). Informed consent was obtained from all individual participants included in the study.

\section{Consent for publication}

Written consent for publication was obtained from the patient.

\section{Competing interests}

The authors declare that they have no competing interests.

\section{References}

1. Prat J; FIGO Committee on Gynecologic Oncology: Staging classification for cancer of the ovary, fallopian tube, and peritoneum. Int J Gynaecol Obstet 124: 1-5, 2014.

2. Jayson GC, Kohn EC, Kitchener HC and Ledermann JA: Ovarian cancer. Lancet 384: 1376-1388, 2014.

3. Goff BA, Mandel LS, Melancon CH and Muntz HG: Frequency of symptoms of ovarian cancer in women presenting to primary care clinics. JAMA 291: 2705-2712, 2004.

4. Kamura T and Jeon JD: Lymph node metastasis in a gynecologic malignancy. Yonsei Med J 43: 783-791, 2002.

5. Rahman M, Nakayama K, Rahman MT, Katagiri H, Ishibashi T and Miyazaki K: Enlarged Virchow's node as an initial complaint of serous ovarian adenocarcinoma. Eur J Gynaecol Oncol 33: 546-548, 2012.

6. Ceccarelli F, Barberi S, Pontesilli A, Zancla S and Ranieri E: Ovarian carcinoma presenting with axillary lymph node metastasis: A case report. Eur J Gynaecol Oncol 32: 237-239, 2011.

7. Cheng B, Lu W, Xiaoyun W, YaXia C and Xie X: Extra-abdominal metastases from epithelial ovarian carcinoma: An analysis of 20 cases. Int J Gynecol Cancer 19: 611-614, 2009.

8. Yang XJ, Zheng FY, Xu YS and Ou RY: Ovarian cancer initially presenting with isolated ipsilateral superficial inguinal lymph node metastasis: A case study and review of the literature. J Ovarian Res 7: 20, 2014.

9. Fagotti A and Scambia G: Counterpoint: Primary Debulking surgery vs neoadjuvant chemotherapy for newly diagnosed advanced ovarian cancer. Oncology (Williston Park) 31: 453, 458, 460-461, 2017.

10. Petrillo M, Zannoni GF, Tortorella L, Pedone Anchora L, Salutari V, Ercoli A, Margariti PA, Scambia G and Fagotti A: Prognostic role and predictors of complete pathologic response to neoadjuvant chemotherapy in primary unresectable ovarian cancer. Am J Obstet Gynecol 211: 632.e1-e8, 2014.

11. Tsilidis KK, Allen NE, Key TJ, Dossus L, Lukanova A, Bakken K, Lund E, Fournier A, Overvad K, Hansen L, et al: Oral contraceptive use and reproductive factors and risk of ovarian cancer in the European prospective investigation into cancer and nutrition. Br J Cancer 105: 1436-1442, 2011.

12. Cormio G, Rossi C, Cazzolla A, Resta L, Loverro G, Greco P and Selvaggi L: Distant metastases in ovarian carcinoma. Int J Gynecol Cancer 13: 125-129, 2003.

13. Cebesoy FB, Balatt O and Aydin A: Virchow's node as a first manifestation of ovarian serous carcinoma: Case report. Eur J Gynaecol Oncol 29: 182-183, 2008.

14. Fanti S, Nanni C, Castellucci P, Farsad M, Rampin L, Gross MD, Mariani G and Rubello D: Supra-clavicular lymph node metastatic spread in patients with ovarian cancer disclosed at 18F-FDG-PET/CT: An unusual finding. Cancer Imaging 6: 20-23, 2006.

15. Hockstein S, Keh P, Lurain JR and Fishman DA: Ovarian carcinoma initially presenting as metastatic axillary lymphadenopathy. Gynecol Oncol 65: 543-547, 1997.

16. Saxena AK, Goyal N, Singhal J and Kumar P: Primary ovarian serous adenocarcinoma with ipsilateral axillary lymph node metastasis: A case report. Indian J Surg Oncol 5: 224-226, 2014.

17. Sibio S, Sammartino P, Accarpio F, Framarino dei Malatesta ML, Biacchi D, Sollazzo BM and Di Giorgio A: Axillary lymph node metastasis as first presentation of peritoneal carcinomatosis from serous papillary ovarian cancer: Case report and review of the literature. Eur J Gynaecol Oncol 35: 170-173, 2014. 
18. Singer C, Blankstein E, Koenigsberg T, Mercado C, Pile-Spellman E and Smith SJ: Mammographic appearance of axillary lymph node calcification in patients with metastatic ovarian carcinoma. AJR Am J Roentgenol 176: 1437-1440, 2001.

19. Aydin C, Unalp HR, Baloğlu A, Inci AG, Yiğit S and Yavuzcan A: Axillary lymph node metastasis from serous ovarian cancer: A case report and review of the literature. Arch Gynecol Obstet 279: 203-207, 2009

20. Ozmen V, Asoglu O, Karanlik H, Cabioglu N, Kecer M and Bakkaloglu H: Primary ovarian cancer presenting with axillary lymph node metastases: A report of two cases. Acta Chir Belg 107: 75-77, 2007.

21. Skagias L, Ntinis A, Vasou O, Kondi-Pafiti A and Politi E: Ovarian carcinoma presenting with axillary lymph node metastasis: A case diagnosed by fine-needle aspiration and brief review of the literature. Diagn Cytopathol 36: 891-893, 2008.
22. Orris BG, Geisler JP and Geisler HE: Ovarian carcinoma metastatic to bilateral axillary lymph nodes. A case report. Eur J Gynaecol Oncol 20: 189-190, 1999.

23. Ang D, Ng KY, Tan HK, Chung AY, Yew BS and Lee VK: Ovarian carcinoma presenting with isolated contralateral inguinal lymph node metastasis: A case report. Ann Acad Med Singapore 36: 427-430, 2007.

24. Zang RY, Zhang ZY, Cai SM, Tang MQ, Chen J and Li ZT: Epithelial ovarian cancer presenting initially with extraabdominal or intrahepatic metastases: A preliminary report of 25 cases and literature review. Am J Clin Oncol 23: 416-419, 2000 . 